\section{Laplacian Pattern Formation}

\section{Tamás Vicsek and János Kertész, Budapest}

(Institut for Technical Physics, HAS)

The formation of snowflakes, viscous fingers and electrodeposits is governed by analogous equations: these structures are all Laplacian patterns. In this paper we review model experiments and computer simulations which are widely used to study diverse morphologies resulting from the motion of unstable interfaces.

Far from equilibrium process resulting in the growth of complex interfacial structures are common phenomena in many fields of science and technology. Examples for the formation of such patterns, among many others, include dendritic solidification when the crystalline phase is growing in an undercooled melt, viscous fingering which can be observed if a less viscous fluid is injected into a more viscous one and electrodeposition of ions onto an electrode. Fig. 1 demonstrates the richness of complex interfaces which are formed under a wide variety of experimental conditions.

The explanation for the apparently analogous behaviour of these different growth processes lies in the equations which describe the motion of the interface. The basic equation is

$$
\nabla^{2} u=0
$$

where $u$ may denote the distribution of pressure (viscous fingering), temperature (crystallization) or electric field (electrodeposition). Equation (1) usually corresponds to some approximations, but it captures the essential physics.

When solving (1) one has to satisfy the appropriate boundary conditions. The normal velocity of the interface, $v_{n}$, is given by

$$
v_{\mathrm{n}}=-k(\nabla u) \mathrm{n},
$$

where $k$ is a constant. The second boundary condition prescribes the value of $u$ on the interface $\Gamma$

$$
u_{\Gamma}=-d_{o} \kappa-\beta v_{n}^{\gamma},
$$

where the capillary length $d_{0}$ is proportional to the surface tension, $\kappa$ denotes the local curvature of the interface and $\gamma$ is an exponent depending on the physical process considered. The first term in the right hand side corresponds to the assumption of local thermodynamic equilibrium, while the second, velocity dependent term with the kinetic coefficient $\beta$ represents the departure from the equilibrium. Since the concentration of diffusing particles is also determined by the Laplace equation (1), growth phenomena described by (1-3) are usually called diffusion-limited or Laplacian processes.
Three main approaches have been developed to treat the problem posed by equations (1-3):

(i) Analytical methods including stability analysis and search for solutions of significantly simplified versions of the original equations have been used by many authors. Groups at University of California, Schlumberger-Doll Research and Ecole Normale Supérieure in Paris have demonstrated the crucial role of anisotropy of the surface tension in pattern formation and the existence of a solvability condition which determines the discrete set of interfacial velocities from which the largest one is selected.

(ii) To study the geometrical properties of very complex patterns it is more effective to use various simulation techniques. The diffusion-limited aggregation (DLA) model introduced by Witten and Sander in 1981 has been shown to capture the most important features of Laplacian pattern formation with noise. In this model randomly walking particles launched from distant points stick to the surface of the growing aggregate whenever they hit it. DLA and its generalizations have contributed to the understanding of diffusion-limited pattern formation by leading to a variety of patterns analogous to those shown in Fig. 1.

(iii) Experiments on pattern formation are usually inexpensive and relatively easy to carry out. There have been a number of experiments recently which have revealed interesting new information about Laplacian growth. In the following we shall discuss a few recent results obtained by the latter two approaches.

A closer look at the structures displayed in Fig. 1 suggests that the patterns can be ordered into specific morphological phases depending on the conditions during the experiments. The overall appearance of the patterns can be quite different: in some cases the interface bounds a region which is irregular but still homogeneous on a length scale comparable with the size of the whole structure (Figs. 1a, d and g).
For another set of the parameter values the patterns have an open branching structure (Figs. $1 \mathrm{~b}$, e and $\mathrm{h}$ ) and can be described in terms of fractal geometry. This is a quite common situation in growth processes and it means that the growing structure is self-similar in a statistical sense and the volume or mass of the region bounded by the interface, $M$, scales with the increasing linear size $R$ of the object in a nontrivial way

$$
M \sim R^{D} .
$$

Here $D$ is called a fractal dimension and is smaller than the Euclidian dimension $d$ of the space the fractal is embedded in. Of course, the above scaling in a physical system holds only for length scales between a lower and an upper cutoff.

What are the most important parameters and under what conditions do they determine the morphological phase to which a given growth process leads? These are the main questions to be answered by the studies of pattern formation. In the past few years much progress has been made in this field and it has turned out that the formation of patterns is determined by a number of important parameters such as anisotropy, noise and driving force.

The complex behaviour leading to the development of structures has its origin in the instability due to the moving boundary condition (2). For example, in a radial Hele-Shaw cell when air (or a less viscous fluid) is injected into a quasi two-dimensional viscous fluid held between glass plates, the growth is driven by the pressure gradient. The so called Saffmann-Taylor or Mullins-Sekerka instability takes place in this system for bubble sizes exceeding a characteristic length depending on the surface tension and the growth velocity. In this case, whenever one part of the interface advances locally faster then the surrounding region the pressure gradient at this protrusion becomes larger in analogy with the increased electric field at the tip of a charged needle. The increased pressure gradient leads to a faster growth of the interface which, in turn, results in a further increment of the gradient. Therefore, the interface becomes unstable against perturbations (noise) and elongated "fingers" grow out of it. The fingers are getting longer and thicker until their tips become too flat to be stable: they split. Under specific conditions (viscous fingering in a random medium or in a non-Newtonian fluid with vanishing surface tension) tip splitting is followed by a competition between the new-born branches. During this process the more advanced, longer fingers screen the shorter ones. Since this pro- 


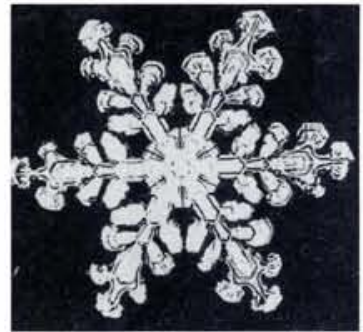

a
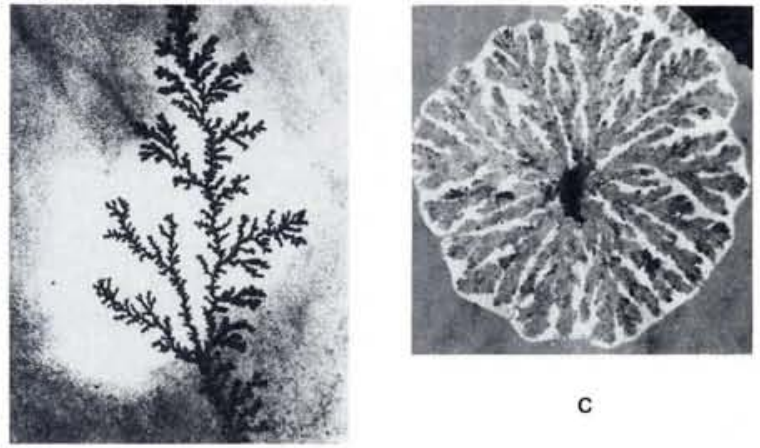

C

b

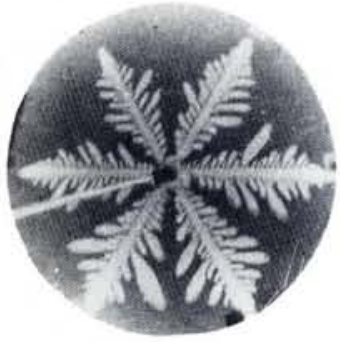

d

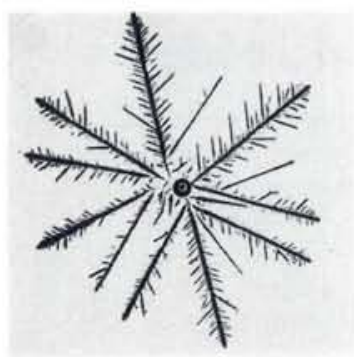

g

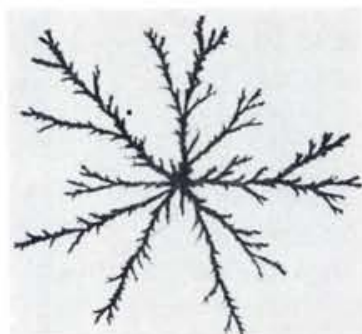

e

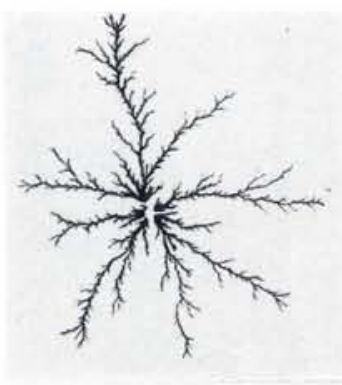

$\mathrm{h}$

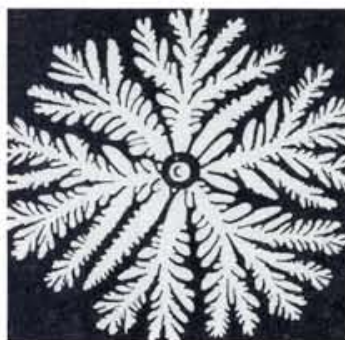

f

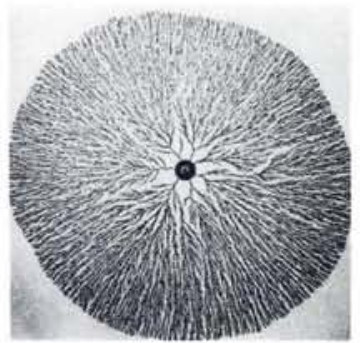

i

Fig. 1 - Laplacian patterns obtained under various experimental conditions. The three major types of structure observed in the experiments on crystallization $(a, b$ and $c)$, viscous fingering ( $d, e$ and $f)$ and electrodeposition of zinc $(g, h$ and i) are grouped into separate columns. (sources: (1a) Ben-Jacob E., Deutscher G., Garik P., Goldenfeld N.D. and Y. Lareah, Phys. Rev. Lett 57 (1986) 1903; (1b) Radnoczy G., Vicsek T., Sander L.M. and Grier D., Phys. Rev. A35 (1987) 4012; (1c) Bentley W.A. and Humpreys W.J., Snow Crystals, (Dover Publications, 1962); (1d) Buka A., Kertész J. and Vicsek T., Nature 323 (1986) 424; (1e) Daccord G., Nittmann J. and H.E. Stanley, Phys. Rev. Lett. 56 (1986) 336; (1f) Ben-Jacob E., Godbey Y., Goldenfeld N., Koplik J., Levine H., Mueller T. and Sander L.M., Phys. Rev. Lett. 55 (1985) 1315; (1g,i) Sawada Y., Dougherty A. and Gollub J.P., Phys. Rev. Lett. 56 (1986) 1260; (1h) Matsushita M., Sano M., Hayakawa Y., Honjo H. and Sawada Y., Phys. Rev. Lett. 53 (1984) 286.

cess is triggered by noise, a random pattern can result from a sequence of such instabilities (Fig. 1e). Similar mechanisms are responsible for the complex geometries developing in other Laplacian processes as well, leading to open, random fractal patterns with $D<d$.

DLA-clusters can be considered as a result of the above described sequence of instabilities: tip splitting resulting in branching and competition between the branches due to screening. The growth process leads to an open ramified structure with a well defined fractal dimensionality ( $D=1.71$ in two dimensions).

If the noise is small and the screening between the branches does not prevail, the fingers emerging from tip splitting keep growing and the space becomes almost filled with the pattern. This phase has a stable envelope and is called dense branching morphology. Its dimensionality is equal to the Euclidian embedding dimension.

Another stable morphological phase develops if there is large enough anisotropy either in the surface tension or in the factor $\beta$ of equation (3) preventing the tips from splitting. Owing to the anisotropy, directions of easy growth are present in which the interface proceeds faster, stabilizing the tips. This is the mechanism governing the regular growth of dendritic crystals like snowflakes.

If diffusion-limited aggregation is simulated on a lattice (e.g. on a square mesh) the anisotropy - which was shown to be relevant for Laplacian growth - is expected to influence the shape of the clusters significantly. In this case the lattice axes represent directions of easy growth. According to the simulations of Paul Meakin from du Pont, Wilmington, however, only extremely large clusters (containing about $10^{6}$ particles) show a tendency towards an overall cross-shaped form. The actual shape of lattice DLA-clusters emerges from an interplay between fluctuation and anisotropy.

The fluctuations in DLA can be controlled by the so called noise reduction method: instead of adding the randomly walking particle to the cluster after it hits a growth site, one keeps counting the trajectories terminating at a given site. Only those sites are added to the cluster at which the counter reaches a prescribed value $m$. Large $m$ corresponds to low level of noise; the $m=1$ is usual DLA.

For a fixed number of particles in the cluster an interesting morphological transition can be observed as a function

a

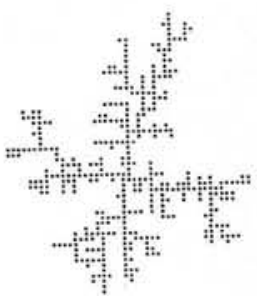

b
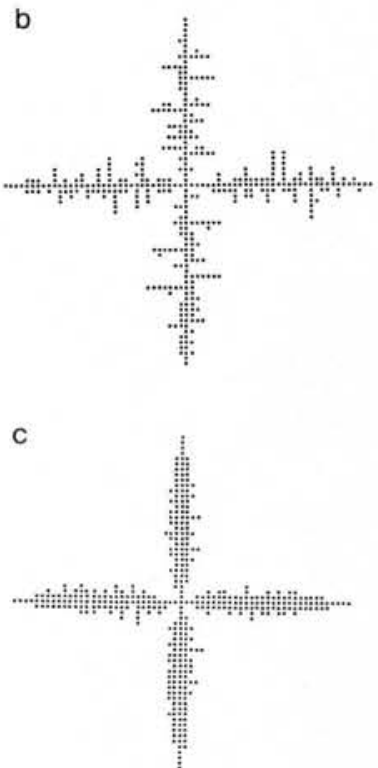

Fig. 2 - The effect of noise reduction on the structure of DLA clusters grown on the square lattice. With the decreasing amount of fluctuations (with growing $\mathrm{m}$ ) the clusters consisting of 400 particles go through a sequence of morphological changes (from Kertész J. and Vicsek T., J. Phys. A 19 (1986) L 257). 


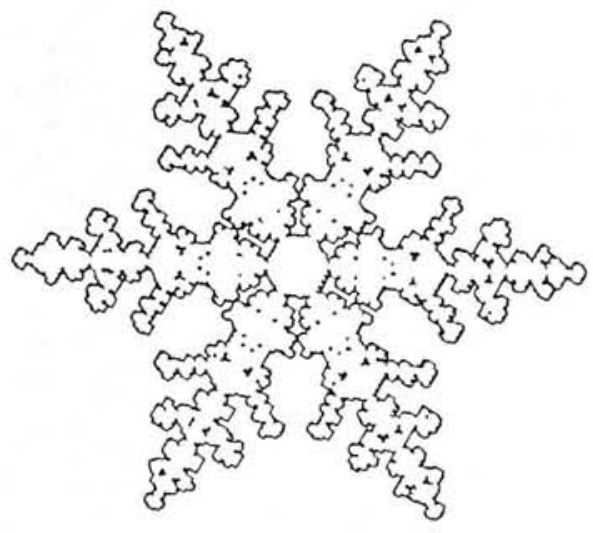

Fig. 3 - This pattern was grown in the computer using the deterministic aggregation model described in the text.

of the noise reduction parameter $m$ (Fig. 2). "Noisy" DLA-clusters grown on the square lattice are open branched structures like off lattice aggregates. If the noise is reduced, the anisotropy due to the grid breaks through and the clusters become cross-shaped with stable tips reminiscent of dendritic crystals. For large values of $m$ even the side branches vanish and one recovers needles growing out of a centre, reflecting the geometry of the lattice. Furthermore, noise reduction is a suitable tool to study the asymptotics of DLA-clusters since the relative weight of fluctuations decreases not only with increasing $m$ but also with increasing size.

The almost perfect symmetry and enormous number of different snowflakes represents a long standing puzzle. It is quite natural to assume that this problem should be treated by a technique with no spatial fluctuations. Indeed, a deterministic growth model designed to provide a good approximation to the equations $(1-3)$ on a triangular lattice is capable of reproducing the basic types

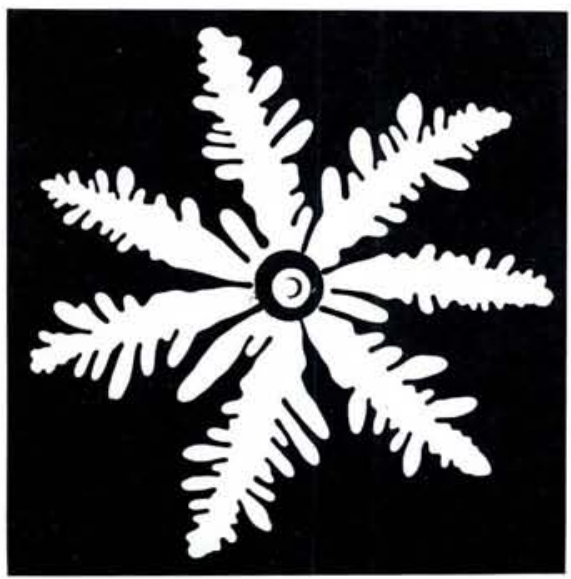

Fig. 4 - Dendritic viscous fingers obtained in a radial Hele-Shaw cell with a nematic liquid crystal between the plates for intermediate pressures of the injected air. For lower pressures the pattern is similar to those obtained in usual isotropic liquids. of snowflake. The process starts with a seed particle. Then the Laplace equation is solved on the triangular grid (providing the sixfold symmetry). The decision whether a particular surface site is filled at a given stage of the growth is made on the basis of comparing the value of the calculated temperature gradient at that site with a time dependent parameter.

Typical patterns obtained by varying a parameter of the deterministic model during the growth are shown in Fig. 3. According to the simulations the great variety of dendritic patterns (like snowflakes) produced by the same solidification process is likely to be due to the temporal changes in such environmental conditions as undercooling or vapour pressure.

Perhaps the most versatile experimental realization of Laplacian pattern formation is viscous fingering. Virtually all the above mentioned important influencing factors can be investigated with relatively small effort. The effect of the driving force is studied by changing the pressure of the less viscous fluid. Noise or anisotropy can be introduced by appropriate preparation of the cell: randomly positioned hindrances are put into the cell or a mesh is grooved onto one of the plates.

What happens if in a Hele-Shaw cell the viscous fluid is inherently anisotropic? Injecting air into a liquid crystal is a realization of this situation. In the case of nematics where the elongated molecules of the liquid have a long range order characterized by a vector called director, an interesting re-entrant morphological transition can be observed as a function of the increasing driving force. In this medium, radial anisotropy is built up by the flow. For low pressure, the shape of the interface is very similar to the usual viscous fingering patterns obtained with isotropic liquids. If higher pressure is applied the tips of the fingers are stable and the bubble becomes dendritic, snowflake-like (Fig. 4). By further increase of the pressure tip, splitting sets in again, probably because the stronger flow results in a disordered, chaotic motion of the director, therefore the effective anisotropy is decreasing and the pattern has a dense branching morphology (DBM) illustrated in Figs. 1a, $\mathrm{d}$ and $\mathrm{g}$.

We have seen that tip splitting with large fluctuations leads to the fractal DLA-structure while without strong noise the nonfractal (DBM) develops. Viscous fingering patterns in a smectic A liquid crystal undergo a morphological phase transition from the DLA to the DBM structure as a function of the pres-
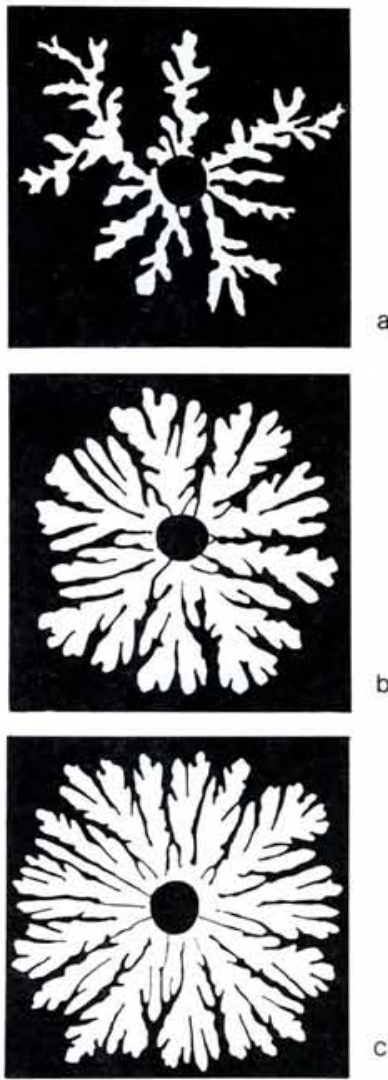

Fig. 5 - On increasing the pressure of injected air in the experiments on viscous fingering in smectics a crossover from a DLA-type fractal interface (a) to a nonfractal, dense branching morphology (c) can be observed (from Horváth V., Kertész J. and Vicsek T., Europhys. Lett. 4 (1987) 1133).

sure of the injected air. The structure of smectics is more ordered than that of nematics: the aligned molecules are arranged into layers which can slip on each other determining this way directions of easy growth of the bubble. The domain structure of the liquid crystal introduces an orientational disorder into the system which leads for low pressures to DLAtype structures. The effective fractal dimension $D_{\text {eff }}$ of the pattern in Fig. $5 a$ is 1.6 , very close to the two-dimensional DLA value. For high pressure, the flow destroys the order of the liquid crystal and we recover the dense branching morphology with $D_{\text {eff }} \cong 2$.

In order to study the effects of anisotropy on the shape of viscous fingers one can use an alternative approach by engraving a mesh on the surface of the plates. This trick was first used by BenJacob and his collaborators to obtain a sequence of morphological changes as a function of the increasing pressure of injected air. Perhaps the simplest kind of anisotropy can be introduced by etching a set of parallel grooves on one of the plates.

The results are quite interesting: the morphological phase diagram (Fig. 6) of 


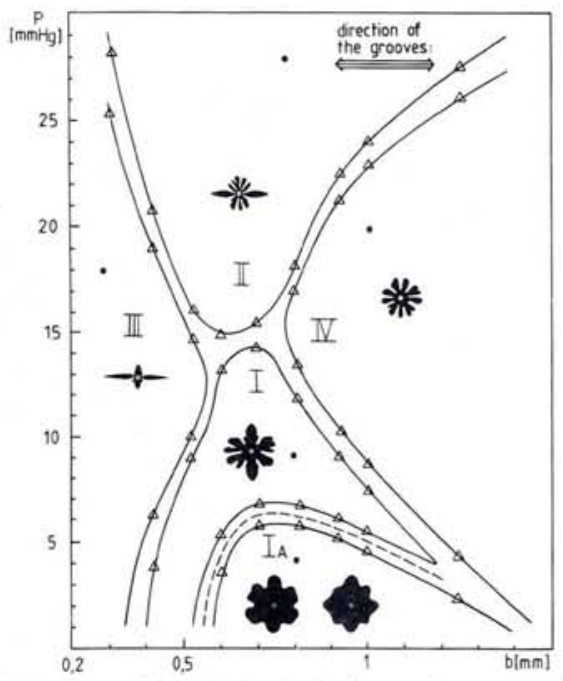

Fig. 6 - Morphological phase diagram of the patterns observed in a uniaxially engraved Hele-Shaw cell (V. Horváth, T. Vicsek and J. Kertész, Phys. Rev. A35 (1987) 2353). the system shows a variety of patterns unexpected for such a type of anisotropy. For example, if the pressure is low, the growth can be stable in the "hard" direction (perpendicular to the grooves) and simultaneously unstable (splitting tips) along the grooves. On increasing the pressure the situation becomes reversed. The effects induced by the competition between the anisotropy and the driving force result in a truly complicated behaviour in this system.

The above simulations and experiments elucidate the crucial role of anisotropy, noise and driving force in the formation of unstable interfaces. The interplay of these factors determines the actual shape of the patterns. Although much progress have been made in the past few years towards understanding the major features of Laplacian growth phenomena we are far from being able to explain complex morphological phase diagrams. The open fundamental problems include the existence of critical parameters, the effect of fluctuations on side branching or the role of temporal fluctuations. Important future work, both experimental and theoretical, is expected in this field.

\section{SUGGESTED READING}

Langer J.S., Rev. Mod. Phys. 52 (1980) 1. Bensimon D., Kadanoff L.P., Liang S., Shraiman B.I. and Tang L., Rev. Mod. Phys. 58 (1986) 977.

On Growth and Form: Fractal and Nonfractal Patterns in Physics, eds. H.E. Stanley and N. Ostrowsky (Martinus Nijhoff, Dordrecht) 1986.

Fractals in Physics, eds. E. Tossatti and L. Pietronero (North-Holland, Amsterdam) 1986.

\section{NORTH-HOLLAND}

\section{Computer Physics Communications \\ Computer Physics Reports}

The International Journal and Program Library Devoted to Computational Physics and Computer Programs in Physics.

* long publishing tradition

* carefully documented Program Library

* 25 free reprints

* no page charges

* fast publication times

This leading international journal publishes descriptions of computer programs in (chemical) physics, letters and papers on computational methods, and the application of computers to physics in several areas Affiliated to this journal is the C.P.C. Program Library, a nonprofit organization which holds and distributes all the programs published in the journal. As of March 1st, 1988 the Program Library is introducing a new on-line information service to users on the computer network.

\section{Editors}

G.H.F. Diercksen (Garching, F.R.G.)

J.W. Eastwood (Abingdon, U.K.)

J.E. Inglesfield (Daresbury, U.K.)

F. James (Geneva, Switzerland)

D.J. Kuck (Urbana, IL, U.S.A.)

D.G. Truhlar (Minneapolis, MN, U.S.A.)

Show Issue

A show issue has been produced which presents a collection of regular articles that cover most of the scope of Computer Physics Communications.
An International Review Journal Devoted to Methods and Algorithms in Computational Physics

* only review journal in the field

* to date 54 high quality reviews published

* practical information on specialist topics

The journal consists of unique issues, each containing a single review devoted to a separate area of computational physics. Special emphasis will be put on a straightforward presentation of the subject with respect to automatic computation.

Editors

G.H.F. Diercksen (Garching, F.R.G.)

F.A. Gianturco (Rome, Italy)

R. Gruber (Lausanne, Switzerland)

J.E. Inglesfield (Daresbury, U.K.)

M.L. Klein (Pittsburgh, PA, U.S.A.)

D.J. Kuck (Urbana, IL, U.S.A.)

C.W. Patterson (Tucson, AZ, U.S.A.)

C. Rebbi (Boston, MA, U.S.A.)

K.-H. Winkler (Los Alamos, NM, U.S.A.)

N.J. Zabusky (Pittsburgh, PA, U.S.A.)

\section{Subscription Information 1988}

Computer Physics Communications:

Vols. 48-51 in 12 issues

Dfl. 1944.00/US \$1050.75

(incl. postage and handling)

Computer Physics Reports:

Vols. 9 and 10 in 12 issues

Dfl. 912.00/US $\$ 493.00$

(incl. postage and handling)
Reduced combined subscription to $\mathrm{CPC}$ and CPR in 24 issues

Dfl. $2556.00 / \mathrm{US} \$ 1381.50$

(incl. postage and handling).

A detailed brochure is available form the publisher.

In case you are interested in obtaining a free sample copy of Computer Physics Communications or Computer Physics Reports, or want to receive a copy of one of the 1988 catalogues, please return the order form to:

North-Holland Physics Publishing P.O. Box 103, 1000 AC Amsterdam THE NETHERLANDS

Attn. E.P.M. Wijnhoven

\section{ORDER FORM}

I would like to receive:

$\square$ the free show issue of Computer Physics Communications

$\square$ a free sample copy of Computer Physics Reports

$\square$ one of your 1988 catalogues. My fields of interest are:

Please send this information to:

Name:

Address: 